\title{
Statistical Properties of the Capacity of Double Nakagami-m Channels for Applications in V2V Dualhop Communication Systems
}

\author{
Gulzaib Rafiq ${ }^{1}$, Bjørn Olav Hogstad ${ }^{2}$ and Matthias Pätzold ${ }^{3}$ \\ 1,3 University of Agder \\ ${ }^{2}$ University of Navarra \\ ${ }^{1,3}$ Norway \\ ${ }^{2}$ Spain
}

\section{Introduction}

The design and analysis of cascaded fading models has been an active area of research in recent years due to its applications in numerous real world scenarios such as keyhole channels (Salo et al., 2006; Zlatanov et al., 2008), and multihop communication systems (Andersen, 2002; Karagiannidis et al., 2007; Talha \& Pätzold, 2007; Velkov et al., 2009). It is shown in (Chizhik et al., 2002; Ercerg et al., 1997) that in the presence of a keyhole, the fading between each transmit and receive antenna pair in a multi-input multi-output (MIMO) system can be characterized using a double ${ }^{1}$ Rayleigh process. Afterwards, this model has been extended to the double Nakagami- $m$ fading model in (Shin \& Lee, 2004). In (Salo et al., 2006), the authors have listed a few real world scenarios which give rise to the keyhole effect. Two such scenarios include diffraction through the street edges in urban microcellular environments (Ercerg et al., 1997) and traversal of the propagation paths through a narrow space for the case when the distance between the rings of scatterers around the transmitter and receiver is large (Gesbert et al., 2002).

Multihop communication systems on the other hand fall under the category of cooperative diversity techniques (Laneman et al., 2004; Sendonaris et al., 2003). In such systems, the wireless nodes (in a cooperative network) assist each other by relaying the information from the source mobile station (SMS) to the destination mobile station (DMS), hence improving the network coverage quite significantly. If however, the wireless nodes are assumed to be moving with relatively high speed, the concept of multihop communication can be applied to vehicle-to-vehicle (V2V) communication systems, where the source vehicle (SV) or the traffic control center (TCS) communicates with a destination vehicle (DV) via relay vehicles (RV). Figure 1 depicts these two scenarios for the case of dualhop communication, where the information from the TCS (or the SV) is received at the DV via an RV. V2V communication has received a lot of attention in recent years due to its applications in traffic safety and road traffic flow (Gradinescu et al., 2007). The statistical analysis of the received signal envelope under non-line-of-sight (NLOS) propagation conditions in an amplify-and-forward based

\footnotetext{
${ }^{1}$ Throughout this chapter, we will refer to a double process as the product of two independent but not necessarily identical processes.
} 
dualhop communication system can be found in (Patel et al., 2006), where the overall channel between the transmitter and the receiver is modeled using a double Rayleigh process. This model is then extended to the double Rice channel model in (Talha \& Pätzold, 2007), by taking the line-of-sight propagation conditions into account. The statistical properties of the capacity of double Rice channels have been analyzed in (Rafiq \& Pätzold, 2008). However, the Nakagami- $m$ process is considered to be a more general channel model as compared to the Rice and Rayleigh channel models. Hence, to generalize all the aforementioned works in the regime of multihop communication, the authors of (Karagiannidis et al., 2007) have presented the statistical analysis of the $N *$ Nakagami- $m$ model (i.e., a product of $N$ Nakagami- $m$ processes). Moreover, second order statistics for the double Nakagami- $m$ process can be found in (Zlatanov et al., 2008). Though a lot of papers have been published in the literature employing the cascaded fading channel model, the statistical properties of the capacity of double Nakagami- $m$ channels have not been investigated so far. Such channels find applications both in V2V communication systems employing dualhop communication, and keyhole channels (Zlatanov et al., 2008).

In this chapter ${ }^{2}$, we have studied the statistical properties of the capacity of double Nakagami- $m$ channels. Specifically, the influence of the severity of fading on the statistical properties of the capacity of double Nakagami- $m$ channels is analyzed. We have derived exact analytical expressions for the probability density function (PDF), the cumulative distribution function (CDF), the level-crossing rate (LCR), and the average duration of fades (ADF) of the channel capacity. Here, the LCR and ADF of the channel capacity are important characteristic quantities which provide insight into the temporal behavior of the channel capacity (Giorgetti et al., 2003), (Hogstad \& Pätzold, 2004). Our analysis has revealed that if the fading severity in one or both links of double Nakagami- $m$ channels decreases (i.e., increasing the value of the severity parameter $m$ in one or both of the cascaded Nakagami- $m$ processes), the mean channel capacity increases, while the ADF of the channel capacity decreases. Moreover, this effect results in an increase in the LCR of the channel capacity at lower signal levels.

\section{The double Nakagami- $m$ channel model}

In this chapter, we have considered Scenario 2 in Fig. 1, where the channel between the SV and the DV via an RV is represented as a concatenation of the SV-RV and RV-DV channels (Patel et al., 2006; Talha \& Pätzold, 2007). Moreover, we have assumed that the fading in the SV-RV link and the RV-DV link is characterized by Nakagami- $m$ processes denoted by $\chi_{1}(t)$ and $\chi_{2}(t)$, respectively. Hence, the overall fading channel describing the SV-DV link is modelled by a double Nakagami- $m$ process given by (Kovacs et al., 2002; Zlatanov et al., 2008)

$$
\Xi(t)=A_{\mathrm{RV}} \chi_{1}(t) \chi_{2}(t)
$$

where $A_{\mathrm{RV}}$ is a real positive constant representing the relay gain. The PDF $p_{\chi_{i}}(z)$ of the Nakagami- $m$ process $\chi_{i}(t)(i=1,2)$ is given by (Nakagami, 1960)

$$
p_{\chi_{i}}(z)=\frac{2 m_{i}^{m_{i}} z^{2 m_{i}-1}}{\Gamma\left(m_{i}\right) \Omega_{i}^{m_{i}}} e^{-\frac{m_{i} z^{2}}{\Omega_{i}}}, \quad z \geq 0
$$

\footnotetext{
${ }^{2}$ The material in this chapter is based on "Statistical Properties of the Capacity of Double Nakagami- $m$ Channels", by Gulzaib Rafiq, Bjørn Olav Hogstad and Matthias Pätzold which appeared in the proceedings of 5th IEEE International Symposium on Wireless Pervasive Computing, ISWPC 2010, Modena, Italy, May 2010. (C) 2010 IEEE.
} 


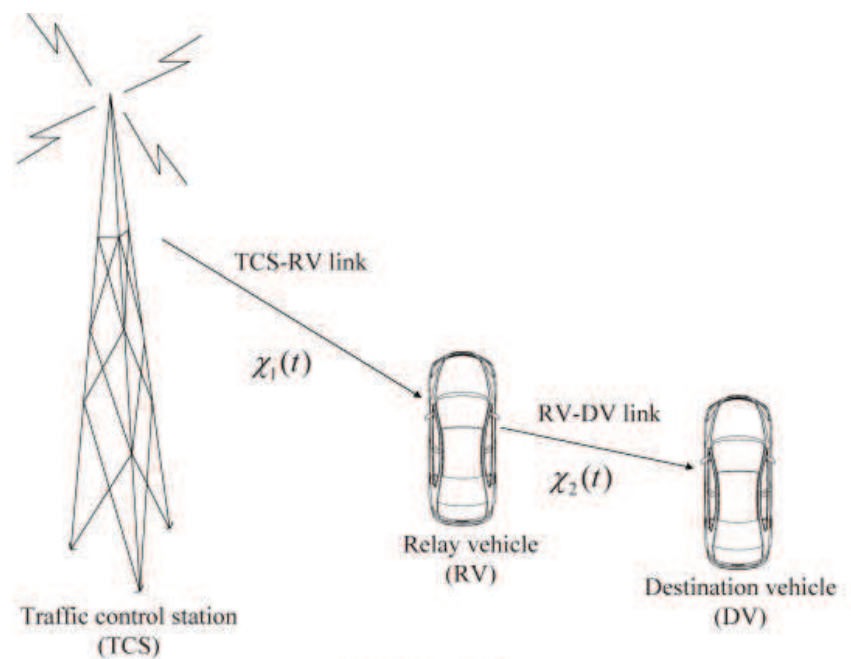

(a) Scenario 1

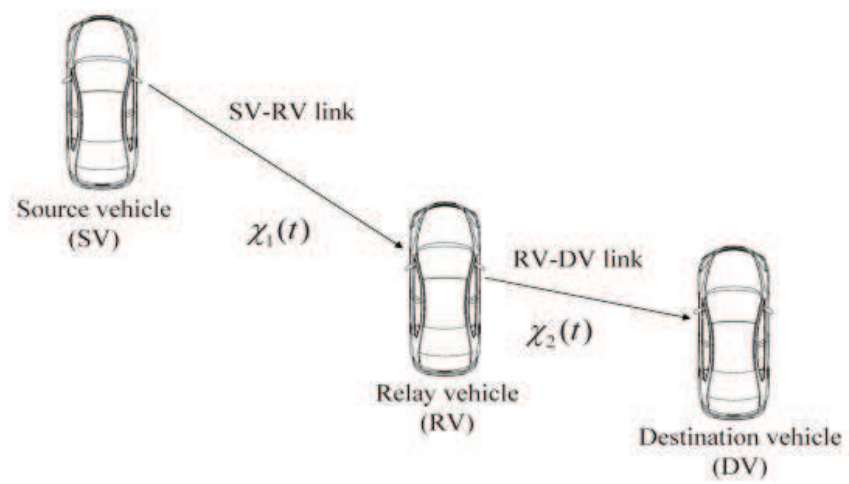

(b) Scenario 2

Fig. 1. The propagation scenarios describing double Nakagami- $m$ fading channels.

where $\Omega_{i}=E\left\{\chi_{i}^{2}(t)\right\}, m_{i}=\Omega_{i}^{2} / \operatorname{Var}\left\{\chi_{i}^{2}(t)\right\}$, and $\Gamma(\cdot)$ represents the gamma function (Gradshteyn \& Ryzhik, 2000). The parameter $m_{i}$ controls the severity of the fading. Increasing the value of $m_{i}$, decreases the severity of fading and vice versa.

The PDF of double Nakagami- $m$ processes $\Xi(t)$ is given by (Karagiannidis et al., 2007)

$$
p_{\Xi}(z)=\frac{4 z^{m_{1}+m_{2}-1}}{\prod_{i=1}^{2} \Gamma\left(m_{i}\right)\left(\dot{\Omega}_{i} / m_{i}\right)^{\left(m_{1}+m_{2}\right) / 2}} K_{m_{1}-m_{2}}\left(2 z \prod_{i=1}^{2} \sqrt{\frac{m_{i}}{\varsigma_{i}}}\right), \quad z \geq 0
$$

where $\Omega_{1}=A_{\mathrm{RV}}^{2} \Omega_{1}, \Omega_{2}=\Omega_{2}$, and $K_{n}(\cdot)$ denotes the modified Bessel function of the second kind of order $n$ (Gradshteyn \& Ryzhik, 2000, Eq. (8.432/1)). In order to derive the expressions for the PDF, CDF, LCR, and ADF of the capacity of double Nakagami- $m$ channels, we need the joint PDF $p_{\Xi^{2} \dot{\Xi}^{2}}(z, \dot{z})$ of the squared process $\Xi^{2}(t)$ and its time derivative $\dot{\Xi}^{2}(t)$, as well as 
the PDF $p_{\Xi^{2}}(z)$ of $\Xi^{2}(t)$. The joint PDF $p_{\Xi^{2} \dot{\Xi}^{2}}(z, \dot{z})$ can be found by following the procedure presented in (Zlatanov et al., 2008) for the joint PDF $p_{\Xi \dot{\Xi}}(z, \dot{z})$ and then by using the concept of transformation of random variables (Papoulis \& Pillai, 2002, Eq. (7-8)), which results in

$$
\begin{aligned}
& p_{\Xi^{2} \dot{\Xi}^{2}}(z, \dot{z})=\frac{1}{4 z} p_{\Xi \dot{\Xi} \dot{\Xi}}\left(\sqrt{z}, \frac{\dot{z}}{2 \sqrt{z}}\right) \\
& =\frac{z^{m_{2}-3 / 2}}{\sqrt{2 \pi}}\left[\prod_{i=1}^{2} \frac{m_{i}^{m_{i}}}{\Omega_{i}^{m_{i}} \Gamma\left(m_{i}\right)}\right] \int_{0}^{\infty} \frac{x^{2 m_{1}-2 m_{2}-1}}{\sqrt{\frac{z \beta_{1}}{x^{2}}+x^{2} \beta_{2}}} e^{-\frac{z m_{2}}{x^{2} \Omega_{2}}} e^{-\left(\frac{z^{2}}{8 z\left(\frac{z \beta_{1}}{x^{2}}+x^{2} \beta_{2}\right)}+\frac{x^{2} m_{1}}{\Omega_{1}}\right)} d x, \\
& z \geq 0,|\dot{z}|<\infty
\end{aligned}
$$

where

$$
\beta_{1}=\frac{\Omega_{1} \pi^{2}}{m_{1}}\left(f_{\max _{1}}^{2}+f_{\max _{2}}^{2}\right)
$$

and

$$
\beta_{2}=\frac{\Omega_{2} \pi^{2}}{m_{2}}\left(f_{\max _{2}}^{2}+f_{\max _{3}}^{2}\right) .
$$

Here, $f_{\max _{1}}, f_{\text {max }_{2}}$, and $f_{\max _{3}}$ represent the maximum Doppler frequencies of the SV, RV, and $\mathrm{DV}$, respectively. The expression for the PDF $p_{\Xi^{2}}(z)$ can be obtained by integrating the joint PDF $p_{\Xi^{2} \dot{\Xi}^{2}}(z, \dot{z})$ over $\dot{z}$. Alternatively, the PDF $p_{\Xi^{2}}(z)$ can also be found from the PDF $p_{\Xi}(z)$ in (3) as follows

$$
\begin{aligned}
p_{\Xi^{2}}(z) & =\frac{1}{2 \sqrt{z}} p_{\Xi}(\sqrt{z}) \\
& =\frac{2 z^{\frac{m_{1}+m_{2}-2}{2}}}{\prod_{i=1}^{2} \Gamma\left(m_{i}\right)\left(\Lambda_{i} / m_{i}\right)^{\left(m_{1}+m_{2}\right) / 2}} K_{m_{1}-m_{2}}\left(2 \sqrt{z} \prod_{i=1}^{2} \sqrt{\frac{m_{i}}{\zeta_{i}}}\right), \quad z \geq 0 .
\end{aligned}
$$

The expressions presented in (4) and (6) will be used in the next section to calculate the PDF and LCR of the channel capacity.

\section{Statistical properties of the capacity of double Nakagami- $m$ channels}

The instantaneous capacity $C(t)$ of double Nakagami- $m$ channels is defined as (Nabar et al., 2004)

$$
C(t)=\frac{1}{2} \log _{2}\left(1+\gamma_{s}|\Xi(t)|^{2}\right)=\frac{1}{2} \log _{2}\left(1+\gamma_{s} \Xi^{2}(t)\right) \quad(\mathrm{bits} / \mathrm{s} / \mathrm{Hz})
$$

where $\gamma_{s}$ denotes the average received signal-to-noise ratio (SNR) at the DV. The factor $1 / 2$ in (7) is due to the fact that the RV in Fig. 1 operates in a half-duplex mode, and hence the signal transmitted from the SV is received at the DV in two time slots. Equation (7) can be considered as a mapping of a random process $\Xi(t)$ to another random process $C(t)$. Hence, the expressions for the statistical properties of the channel capacity $C(t)$ can be found by using the results for the statistical properties of the process $\Xi(t)$ obtained in the previous section. The PDF $p_{C}(r)$ of the channel capacity $C(t)$ can be found in closed form with the help of the PDF 
$p_{\Xi^{2}}(z)$ and by applying the concept of transformation of random variables (Papoulis \& Pillai, 2002, Eq. (7-8)) as

$$
\begin{aligned}
p_{C}(r)= & \left(\frac{2^{2 r+1} \ln (2)}{\gamma_{s}}\right) p_{\Xi^{2}}\left(\frac{2^{2 r}-1}{\gamma_{s}}\right) \\
= & \frac{2^{2 r+2} \ln (2)\left(\left(2^{2 r}-1\right) / \gamma_{s}\right)^{\left(m_{1}+m_{2}\right) / 2}}{\left(2^{r}-1\right) \prod_{i=1}^{2} \Gamma\left(m_{i}\right)\left(\Omega_{i} / m_{i}\right)^{\left(m_{1}+m_{2}\right) / 2}} K_{m_{1}-m_{2}}\left(2 \sqrt{\frac{2^{2 r}-1}{\gamma_{s}}} \prod_{i=1}^{2} \sqrt{\frac{m_{i}}{\Omega_{i}}}\right), r \geq 0 .
\end{aligned}
$$

The CDF $F_{C}(r)$ of the channel capacity $C(t)$ can now be derived by integrating the PDF $p_{C}(r)$ and by making the use of relationships in (Gradshteyn \& Ryzhik, 2000, Eq. (9.34/3)) and (Adamchik \& Marichev, 1990, Eq. (26)) as

$$
\begin{aligned}
F_{C}(r) & =\int_{0}^{r} p_{C}(x) d x \\
& =\frac{1}{\prod_{i=1}^{2} \Gamma\left(m_{i}\right)} G_{1,3}^{2,1}\left[\frac{2^{2 r}-1}{\gamma_{s}} \prod_{i=1}^{2}\left(\frac{m_{i}}{\Omega_{i}}\right) \mid \begin{array}{c}
1 \\
m_{1}, m_{2}, 0
\end{array}\right], \quad r \geq 0
\end{aligned}
$$

where G[·] denotes the Meijer's G-function (Gradshteyn \& Ryzhik, 2000, Eq. (9.301)). The LCR $N_{C}(r)$ of the channel capacity describes the average rate of up-crossings (or down-crossings) of the capacity through a certain threshold level $r$. In order to find the LCR $N_{C}(r)$, we first need to find the joint PDF $p_{C \dot{C}}(z, \dot{z})$ of $C(t)$ and its time derivative $\dot{C}(t)$. The joint PDF $p_{C \dot{C}}(z, \dot{z})$ can be obtained by using the joint PDF $p_{\Xi^{2} \dot{\Xi}^{2}}(z, \dot{z})$ given in (4) as

$$
\begin{aligned}
& p_{C \dot{C}}(z, \dot{z})=\left(\frac{2^{2 z+1} \ln (2)}{\gamma_{s}}\right)^{2} p_{\Xi^{2} \dot{\Xi}^{2}}\left(\frac{2^{2 z}-1}{\gamma_{s}}, \frac{2 \dot{z} \ln (2)}{\gamma_{s} / 2^{2 z}}\right) \\
&= \frac{\left(2^{2 z+1} \ln (2)\right)^{2}\left(2^{2 z}-1\right)^{m_{2}-\frac{3}{2}}}{\sqrt{2 \pi \gamma_{s}} \gamma_{s}^{m_{2}}\left[\prod_{i=1}^{2}\left(\frac{\Omega_{i}}{m_{i}}\right)^{m_{i}} \Gamma\left(m_{i}\right)\right]} \int_{0}^{\infty} \frac{x^{2 m_{1}-2 m_{2}-1}}{\sqrt{\frac{\left(2^{2 z}-1\right) \beta_{1}}{\gamma_{s} x^{2}}+x^{2} \beta_{2}}} e^{-\frac{\left(2^{2 z+1} \ln (2) \dot{z}\right)^{2}}{8 \gamma_{s}\left(2^{2 z}-1\right)\left(\frac{\left(2^{2 z}-1\right) \beta_{1}}{\gamma_{s} x^{2}}+x^{2} \beta_{2}\right)}} \\
& \times e^{-\left(\frac{x^{2} m_{1}}{\Omega_{1}}+\frac{\left(2^{2 z}-1\right) m_{2}}{\gamma_{s} \Omega^{2} \Omega_{2}}\right)} d x
\end{aligned}
$$

for $z \geq 0$ and $|\dot{z}|<\infty$. Finally, the LCR $N_{C}(r)$ can be found as follows

$$
\begin{aligned}
N_{C}(r) & =\int_{0}^{\infty} \dot{z} p_{C \dot{C}}(r, \dot{z}) d \dot{z} \\
& =\sqrt{\frac{8}{\pi}}\left(\frac{2^{2 r}-1}{\gamma_{s}}\right)^{m_{2}-\frac{1}{2}}\left[\prod_{i=1}^{2} \frac{m_{i}^{m_{i}}}{\Omega_{i}^{m_{i}} \Gamma\left(m_{i}\right)}\right] \int_{0}^{\infty} e^{-\frac{\left(2^{2 r}-1\right) m_{2}}{\gamma_{s} x^{2} \Omega_{2}}} \frac{\sqrt{\frac{\left(2^{2 r}-1\right) \beta_{1}}{\gamma_{s} x^{2}}+x^{2} \beta_{2}}}{x^{1+2 m_{2}-2 m_{1}}} e^{-\frac{x^{2} m_{1}}{\Omega_{1}}} d x
\end{aligned}
$$

for $r \geq 0$. The ADF $T_{C}(r)$ of the channel capacity $C(t)$ denotes the average duration of time over which the capacity is below a given level $r$ (Hogstad \& Pätzold, 2004; 2007). The ADF 
$T_{C}(r)$ of the channel capacity can be expressed as (Hogstad \& Pätzold, 2007)

$$
T_{C}(r)=\frac{F_{C}(r)}{N_{C}(r)}
$$

where $F_{C}(r)$ and $N_{C}(r)$ are given by (9) and (11), respectively.

\section{Statistical properties of the capacity of double rayleigh channels}

The double Rayleigh channel follows as a special case of the double Nakagami- $m$ channel when $m_{i}=1(i=1,2)$. Hence, by letting $m_{i}=1(i=1,2)$ in (8), (9), and (11), the PDF, CDF, and LCR of the capacity of double Rayleigh channels can be expressed as

$$
\begin{gathered}
\left.p_{C}(r)\right|_{m_{i}=1}=\frac{2^{2 r} \ln (2)}{\gamma_{s} \sigma_{2}^{2} \sigma_{\mathrm{RV}}^{2}} K_{0}\left(\sqrt{\frac{2^{2 r}-1}{\gamma_{s} \sigma_{2}^{2} \sigma_{\mathrm{RV}}^{2}}}\right), r \geq 0 \\
\left.F_{C}(r)\right|_{m_{i}=1}=1-\sqrt{\frac{2^{2 r}-1}{\gamma_{s} \sigma_{2}^{2} \sigma_{\mathrm{RV}}^{2}}} K_{1}\left(\sqrt{\frac{2^{2 r}-1}{\gamma_{s} \sigma_{2}^{2} \sigma_{\mathrm{RV}}^{2}}}\right), \quad r \geq 0
\end{gathered}
$$

and

$$
\left.N_{C}(r)\right|_{m_{i}=1}=\frac{\sqrt{2^{2 r}-1}}{\sqrt{2 \pi \gamma_{s}} \sigma_{2}^{2} \sigma_{\mathrm{RV}}^{2}} \int_{0}^{\infty} \sqrt{\beta_{2}+\beta_{1}\left(\frac{2^{r}-1}{x^{4} \gamma_{s}}\right)} e^{-\frac{2^{2 r}-1}{2 \sigma_{2}^{2} \gamma_{s} x^{2}}} e^{-\frac{x^{2}}{2 \sigma_{\mathrm{RV}}^{2}}} d y, \quad r \geq 0
$$

respectively. The ADF of the capacity $C(t)$ of double Rayleigh channels can be found using (12), (14), and (15). In (13)-(15), $\sigma_{\mathrm{RV}}^{2}=A_{\mathrm{RV}}^{2} \sigma_{1}^{2}$ and $\sigma_{i}^{2}(i=1,2)$ represent the variances of the underlying Gaussian processes in the corresponding Rayleigh processes $\left.\chi_{i}(t)\right|_{m_{i}=1}(i=1,2)$.

\section{Numerical results}

In this section, we will discuss the analytical results obtained in the previous section. The validity of the theoretical results is confirmed with the help of simulations. For comparison purposes, we have also shown the results for double Rayleigh channels, which represent a special case of double Nakagami- $m$ channels. In order to generate Nakagami- $m$ processes $\chi_{i}(t)$, we have used the following relationship (Yacoub et al., 1999)

$$
\chi_{i}(t)=\sqrt{\sum_{l=1}^{2 \times m_{i}} \mu_{i, l}^{2}(t)}
$$

where $\mu_{i, l}(t) \quad\left(l=1,2, \ldots, 2 m_{i} ; i=1,2\right)$ are the underlying independent and identically distributed (i.i.d.) Gaussian processes, and $m_{i}$ is the parameter of the Nakagami- $m$ distribution associated with the $i$ th link of the dualhop communication systems. The Gaussian processes $\mu_{i, l}(t)$, each with zero mean and variances $\sigma_{0}^{2}$, were simulated using the sum-of-sinusoids model (Pätzold, 2002). The model parameters were computed using the generalized method of exact Doppler spread (GMEDS 1 ) (Pätzold et al., 2009). The number of sinusoids for the generation of Gaussian processes $\mu_{i, l}(t)$ was chosen to be $N=29$. The parameter $\Omega_{i}$ was chosen to be equal to $2 m_{i} \sigma_{0}^{2}$. Unless stated otherwise, the values of the maximum Doppler frequencies $f_{\max _{1}}, f_{\max _{2}}$, and $f_{\max _{3}}$ were taken to be 0,91 , and $125 \mathrm{~Hz}$, respectively. The SNR 
$\gamma_{s}$ was set to $15 \mathrm{~dB}$. The parameters $A_{\mathrm{RV}}$ and $\sigma_{0}$ were chosen to be unity. Finally, using (16), (1), and (7), the simulation results for the statistical properties of the channel capacity were found. The PDF and CDF of the channel capacity of double Nakagami- $m$ channels are presented in Figs. 2 and 3, respectively. Both figures illustrate the fact that increasing the value of the severity parameter $m_{i}$ (i.e., a decrease in the level of the severity of fading) in one or both links of the double Nakagami- $m$ channels results in an increase in the mean channel capacity. This result is specifically presented in Fig. 4, where the mean channel capacity is studied for different values of the severity parameter $m_{i}(i=1,2)$. It can also be seen that double Rayleigh channels $\left(m_{i}=1 ; i=1,2\right)$ have a lower mean channel capacity as compared to the mean channel capacity of double Nakagami- $m$ channels $\left(m_{i}=2 ; i=1,2\right)$. Moreover, it can also be observed from Figs. 2 and 3 that increasing the value of the severity parameter $m_{i}$ decreases the variance of the channel capacity.

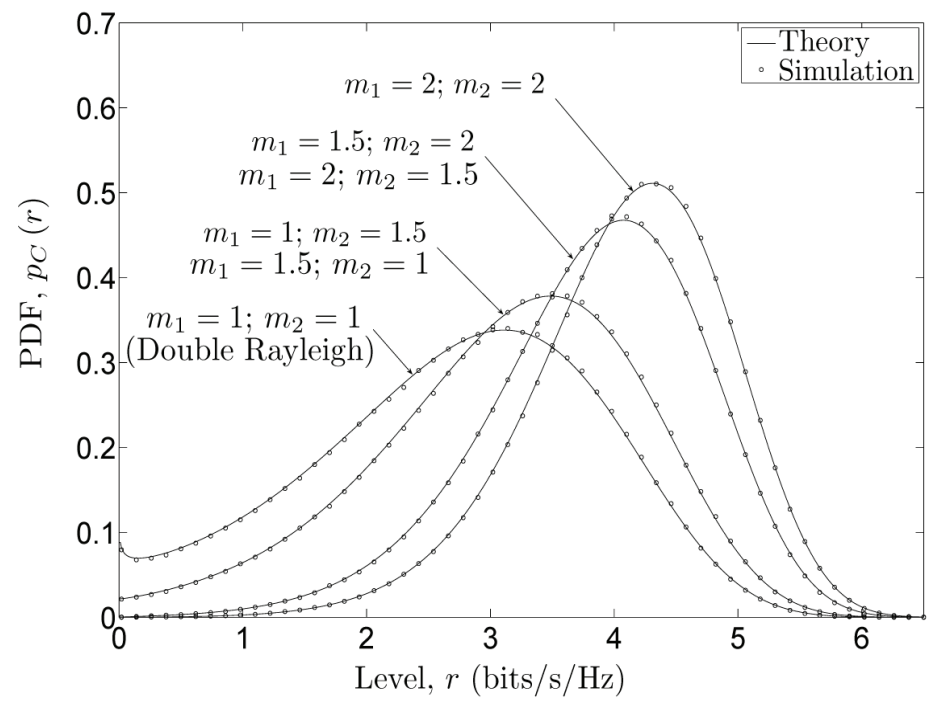

Fig. 2. The PDF $p_{C}(r)$ of the capacity of double Nakagami- $m$ channels.

Figure 5 presents the LCR $N_{C}(r)$ of the capacity $C(t)$ of double Nakagami- $m$ channels. It is observed that an increase in the level of severity of fading in one or both links of double Nakagami- $m$ channels increases the LCR $N_{C}(r)$ of the channel capacity at low levels $r$. Hence, at low levels $r$, the LCR $N_{C}(r)$ of the capacity of double Rayleigh channels $\left(m_{i}=1 ; i=1,2\right)$ is higher as compared to that of double Nakagami- $m$ channels $\left(m_{i}=2 ; i=1,2\right)$. However, the converse statement is true for higher levels $r$. The ADF of the capacity of double Nakagami- $m$ channels is shown in Fig. 6. It is evident from this figure that the ADF of the capacity decreases with an increase in the value of the severity parameter $m_{i}(i=1,2)$.

Figures 7 and 8 study the influence of the maximum Doppler frequencies of the RV and the DV on the LCR and ADF of the channel capacity. It can clearly be observed in Figs. 7 and 8 that the LCR and ADF are strongly dependent on the Doppler frequencies of the RV and the DV. This means that the mobility of the RV and the DV has a significant influence on the LCR and $\mathrm{ADF}$ of the channel capacity. It is observed that increasing the maximum Doppler frequencies 


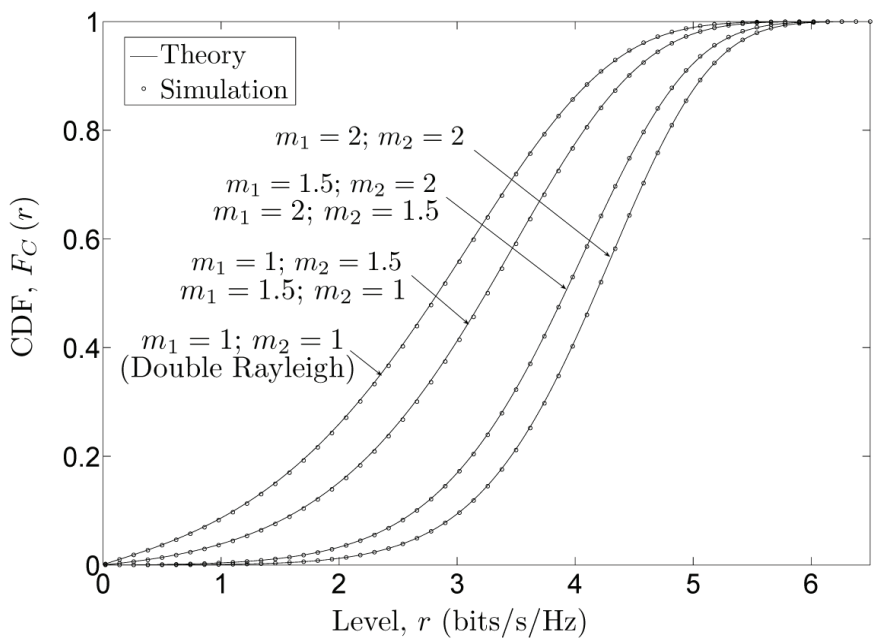

Fig. 3. The CDF $F_{C}(r)$ of the capacity of double Nakagami- $m$ channels.

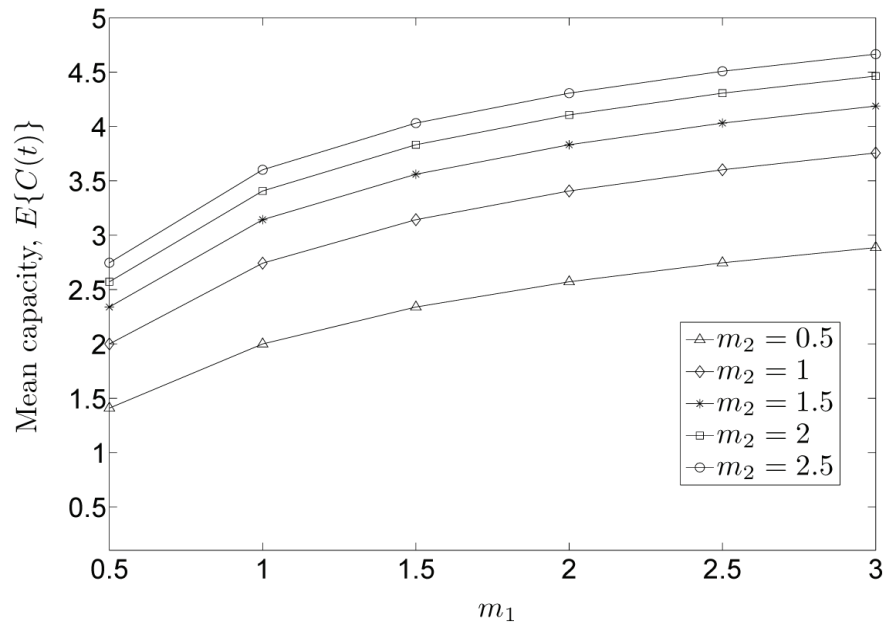

Fig. 4. The mean channel capacity of double Nakagami- $m$ channels for different levels of fading severity.

$f_{\max _{2}}$ and $f_{\max _{3}}$ results in a significant increase in the LCR. However, the ADF decreases by increasing the maximum Doppler frequencies of the RV and the DV.

\section{Conclusion}

This Chapter presents the derivation of exact analytical expressions for the statistical properties of the capacity of double Nakagami- $m$ channels, which finds applications in V2V communication systems employing dualhop communication, and keyhole channels. We have studied the influence of the severity of fading on the PDF, CDF, LCR, and ADF of the channel 


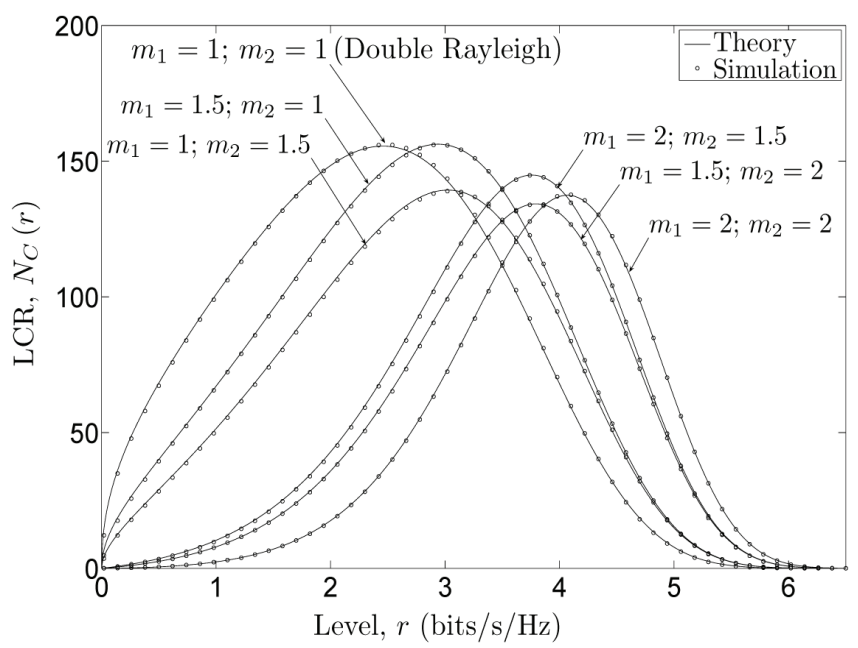

Fig. 5. The LCR $N_{C}(r)$ of the capacity of double Nakagami- $m$ channels.

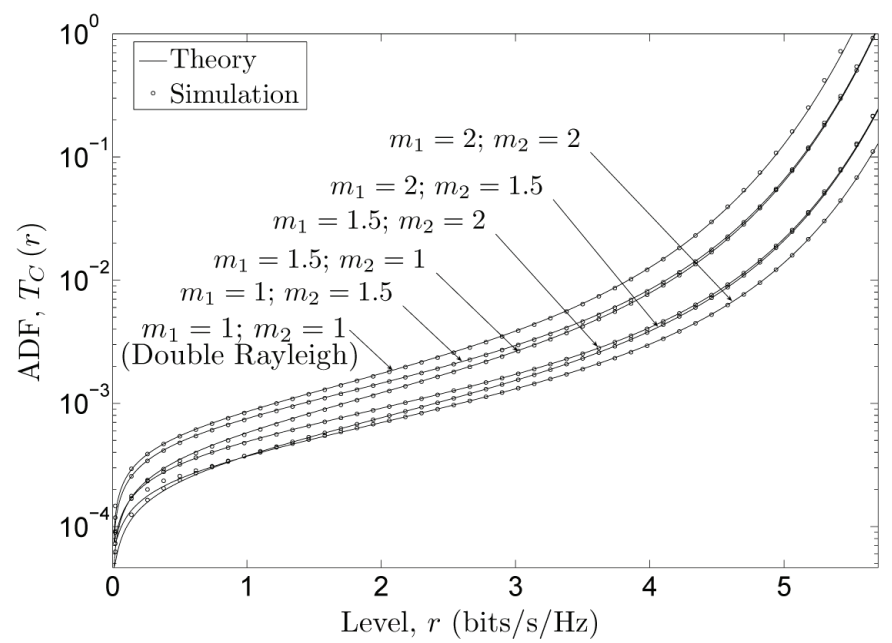

Fig. 6. The ADF $T_{C}(r)$ of the capacity of double Nakagami- $m$ channels.

capacity. It is observed that an increase in the severity of fading in one or both links of double Nakagami- $m$ channels decreases the mean channel capacity, while it results in an increase in the ADF of the channel capacity. Moreover, at lower signal levels, this effects increases the LCR of the channel capacity. Results also show that the mobility of the RV and DV has a significant influence on the LCR and ADF of the channel capacity. Specifically, an increase in the maximum Doppler frequencies of the RV and DV increases the LCR, while it has an opposite influence on the ADF of the channel capacity. The presented exact results are validated with the help of simulations, whereby a very good fitting is observed. 


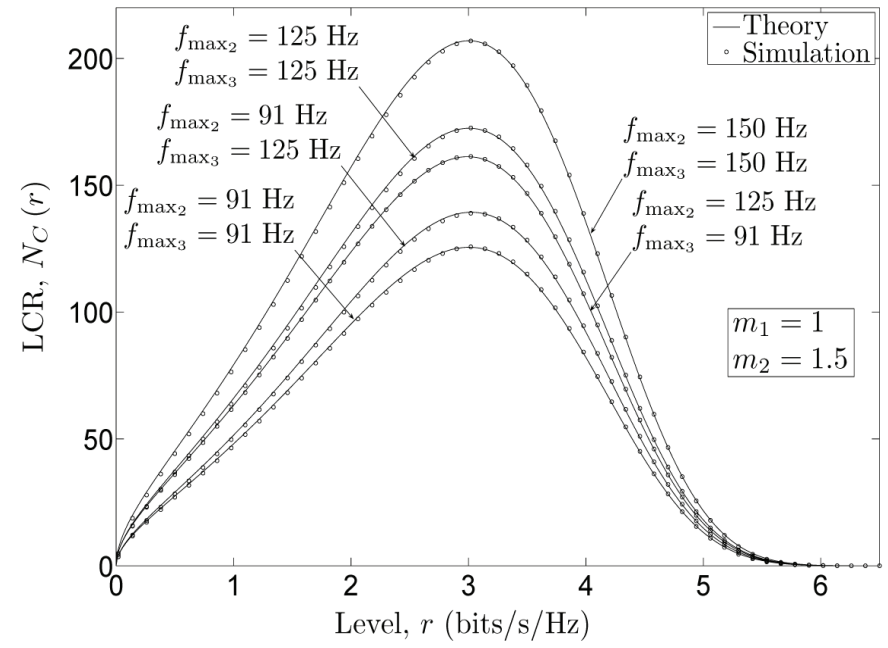

Fig. 7. The LCR $N_{C}(r)$ of the capacity of double Nakagami- $m$ channels.

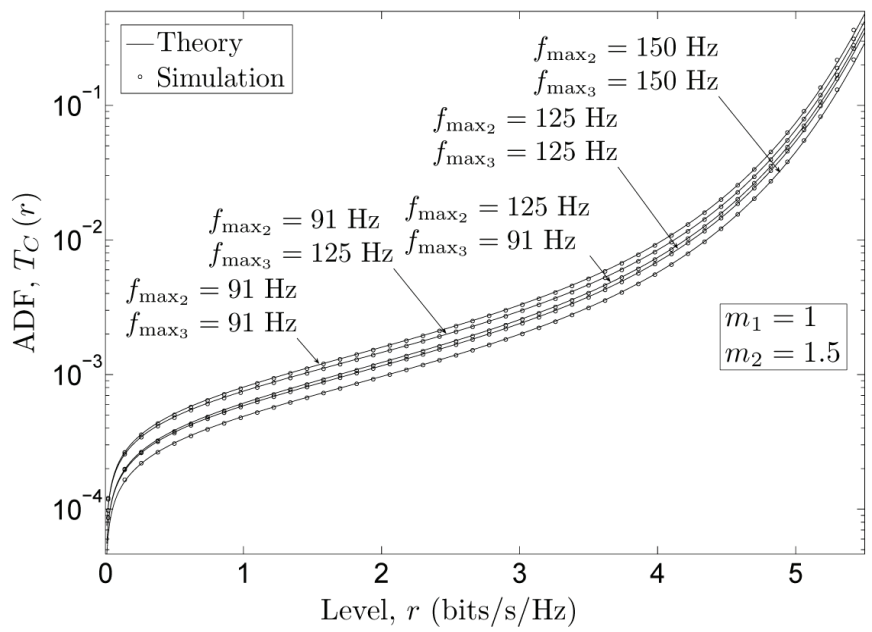

Fig. 8. The ADF $T_{C}(r)$ of the capacity of double Nakagami- $m$ channels.

\section{Acknowledgment}

The contribution of G. Rafiq and Prof. M. Pätzold in this chapter was partially supported by the Research Council of Norway (NFR) through the project 176773/S10 entitled "Optimized Heterogeneous Multiuser MIMO Networks - OptiMO".

The contribution of Dr. B. O. Hogstad was supported in part by the Basque Government through the MIMONET project (PC2009-27B), and by the Spanish Ministry of Science and Innovation through the projects COSIMA (TEC2010-19545-C04-02) and COMONSENS (CSD2008-00010). 


\section{References}

Adamchik, V. S. \& Marichev, O. I. (1990). The algorithm for calculating integrals of hypergeometric type functions and its realization in REDUCE system, Proc. Int. Symp. Symbolic and Algebraic Computation, ISSAC '90, Tokyo, Japan, pp. 212-224.

Andersen, J. B. (2002). Statistical distributions in mobile communications using multiple scattering, Proc. 27th URSI General Assembly, Maastricht, Netherlands.

Chizhik, D., Foschini, G. J., Gans, M. J. \& Valenzuela, R. A. (2002). Keyholes, correlations, and capacities of multielement transmit and receive antennas, IEEE Trans. Wireless Commun. 1(2): 361-368.

Ercerg, V., Fortune, S. J., Ling, J., Rustako Jr., A. J. \& Valenzuela, R. A. (1997). Comparison of a computer-based propagation prediction tool with experimental data collected in urban microcellular environment, IEEE J. Select. Areas Commun. 15(4): 677-684.

Gesbert, D., Bölcskei, H., Gore, D. A. \& Paulraj, A. J. (2002). Outdoor MIMO wireless channels: Models and performance prediction, IEEE Trans. Commun. 50(12): 1926-1934.

Giorgetti, A., Smith, P. J., Shafi, M. \& Chiani, M. (2003). MIMO capacity, level crossing rates and fades: The impact of spatial/temporal channel correlation, J. Commun. Net. 5(2): 104-115.

Gradinescu, V., Gorgorin, C., Diaconescu, R. \& Cristea, V. (2007). Adaptive traffic lights using car-to-car communication, Proc. 65th IEEE Semiannual Vehicular Technology Conference, IEEE VTC 2007-Spring, Dublin, Ireland, pp. 21-25.

Gradshteyn, I. S. \& Ryzhik, I. M. (2000). Table of Integrals, Series, and Products, 6th edn, Academic Press.

Hogstad, B. O. \& Pätzold, M. (2004). Capacity studies of MIMO models based on the geometrical one-ring scattering model, Proc. 15th IEEE Int. Symp. on Personal, Indoor and Mobile Radio Communications, PIMRC 2004, Vol. 3, Barcelona, Spain, pp. 1613-1617.

Hogstad, B. O. \& Pätzold, M. (2007). Exact closed-form expressions for the distribution, level-crossing rate, and average duration of fades of the capacity of MIMO channels, Proc. 65th Semiannual Vehicular Technology Conference, IEEE VTC 2007-Spring, Dublin, Ireland, pp. 455-460.

Karagiannidis, G. K., Sagias, N. C. \& Mathiopoulos, P. T. (2007). N*Nakagami: A novel stochastic model for cascaded fading channels, IEEE Trans. Commun. 55(8): 1453-1458.

Kovacs, I. Z., Eggers, P. C. F., Olesen, K. \& Petersen, L. G. (2002). Investigations of outdoor-to-indoor mobile-to-mobile radio communication channels, Proc. IEEE 56th Veh. Technol. Conf., VTC'02-Fall, Vancouver BC, Canada, pp. 430-434.

Laneman, J. N., Tse, D. N. C. \& Wornell, G. W. (2004). Cooperative diversity in wireless networks: Efficient protocols and outage behavior, IEEE Trans. Inform. Theory 50(12): 3062-3080.

Nabar, R. U., Bölcskei, H. \& Kneubühler, F. W. (2004). Fading relay channels: Performance limits and space-time signal design, IEEE J. Select. Areas Commun. 22: 1099-1109.

Nakagami, M. (1960). The $m$-distribution: A general formula of intensity distribution of rapid fading, in W. G. Hoffman (ed.), Statistical Methods in Radio Wave Propagation, Oxford, UK: Pergamon Press.

Papoulis, A. \& Pillai, S. U. (2002). Probability, Random Variables and Stochastic Processes, 4th edn, New York: McGraw-Hill. 
Patel, C. S., Stüber, G. L. \& Pratt, T. G. (2006). Statistical properties of amplify and forward relay fading channels, IEEE Trans. Veh. Technol. 55(1): 1-9.

Pätzold, M. (2002). Mobile Fading Channels, Chichester: John Wiley \& Sons.

Pätzold, M., Wang, C.-X. \& Hogstad, B. (2009). Two new methods for the efficient generation of multiple uncorrelated Rayleigh fading waveforms, IEEE Trans. Wireless Commun. 8(6): 3122Ü-3131.

Rafiq, G. \& Pätzold, M. (2008). On the statistical properties of the capacity of amplify-and-forward channels under LOS conditions, Proc. IEEE 11th Int. Conf. Communication Systems, IEEE ICCS 2008, Guangzhou, China, pp. 1614-1619.

Salo, J., El-Sallabi, H. M. \& Vainikainen, P. (2006). Impact of double-Rayleigh fading on system performance, Proc. 1st IEEE Int. Symp. on Wireless Pervasive Computing, ISWPC 2006, Phuket, Thailand.

Sendonaris, A., Erkip, E. \& Aazhang, B. (2003). User cooperation diversity—Part I: System description, IEEE Trans. Commun. 51(11): 1927-1938.

Shin, H. \& Lee, J. H. (2004). Performance analysis of space-time block codes over keyhole Nakagami-m fading channels, IEEE Trans. Veh. Technol. 53(2): 351-362.

Talha, B. \& Pätzold, M. (2007). On the statistical properties of double Rice channels, Proc. 10th International Symposium on Wireless Personal Multimedia Communications, WPMC 2007, Jaipur, India, pp. 517Ü-522.

Velkov, Z. H., Zlatanov, N. \& Karagiannidis, G. K. (2009). On the second order statistics of the multihop Rayleigh fading channel, IEEE Trans. Commun. 57(6): 1815-1823.

Yacoub, M. D., Bautista, J. E. V. \& de Rezende Guedes, L. G. (1999). On higher order statistics of the Nakagami-m distribution, IEEE Trans. Veh. Technol. 48(3): 790-794.

Zlatanov, N., Velkov, Z. H. \& Karagiannidis, G. K. (2008). Level crossing rate and average fade duration of the double Nakagami-m random process and application in MIMO keyhole fading channels, IEEE Communications Letters 12(11): 822-824. 


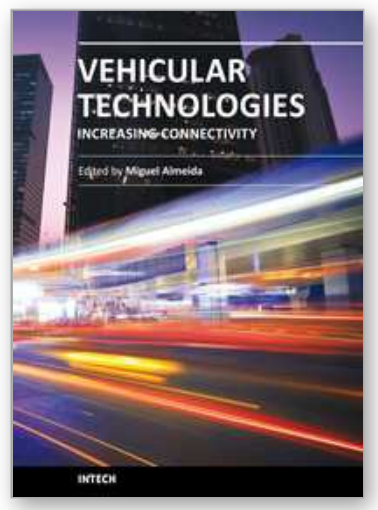

\author{
Vehicular Technologies: Increasing Connectivity \\ Edited by Dr Miguel Almeida
}

ISBN 978-953-307-223-4

Hard cover, 448 pages

Publisher InTech

Published online 11, April, 2011

Published in print edition April, 2011

This book provides an insight on both the challenges and the technological solutions of several approaches, which allow connecting vehicles between each other and with the network. It underlines the trends on networking capabilities and their issues, further focusing on the MAC and Physical layer challenges. Ranging from the advances on radio access technologies to intelligent mechanisms deployed to enhance cooperative communications, cognitive radio and multiple antenna systems have been given particular highlight.

\title{
How to reference
}

In order to correctly reference this scholarly work, feel free to copy and paste the following:

Gulzaib Rafiq, Bjørn Olav Hogstad and Matthias Pätzoldt (2011). Statistical Properties of the Capacity of Double Nakagami-m Channels for Applications in V2V Dualhop Communication Systems, Vehicular Technologies: Increasing Connectivity, Dr Miguel Almeida (Ed.), ISBN: 978-953-307-223-4, InTech, Available from: http://www.intechopen.com/books/vehicular-technologies-increasing-connectivity/statistical-properties-ofthe-capacity-of-double-nakagami-m-channels-for-applications-in-v2v-dualhop

\section{INTECH}

open science | open minds

\section{InTech Europe}

University Campus STeP Ri

Slavka Krautzeka 83/A

51000 Rijeka, Croatia

Phone: +385 (51) 770447

Fax: +385 (51) 686166

www.intechopen.com

\section{InTech China}

Unit 405, Office Block, Hotel Equatorial Shanghai

No.65, Yan An Road (West), Shanghai, 200040, China

中国上海市延安西路65号上海国际贵都大饭店办公楼 405 单元

Phone: +86-21-62489820

Fax: +86-21-62489821 
(C) 2011 The Author(s). Licensee IntechOpen. This chapter is distributed under the terms of the Creative Commons Attribution-NonCommercialShareAlike-3.0 License, which permits use, distribution and reproduction for non-commercial purposes, provided the original is properly cited and derivative works building on this content are distributed under the same license. 